\title{
Efeitos na saúde de populações rurais expostas cronicamente a baixas doses de organofosforados: revisão sistemática
}

\author{
Health effects of rural populations chronically exposed to low doses of organophosphates: \\ systematic review \\ Efectos sobre la salud de las poblaciones rurales crónicamente expuestas a bajas dosis de \\ organofosforados: revisión sistemática
}

Fábio Ricardo Wittke

ORCID: https://orcid.org/0000-0003-3412-3538

Universidade do Vale do Taquari, Brasil

E-mail: fabio.wittke@universo.univates.br

Jordana Kich

ORCID: https://orcid.org/0000-0002-8096-1770

Universidade do Vale do Taquari, Brasil

E-mail: jordana.kich@ universo.univates.br

Claudete Rempel

ORCID: https://orcid.org/0000-0001-8573-0237 Universidade do Vale do Taquari, Brasil

E-mail: crempel@univates.br

Laura Gaspary

ORCID: https://orcid.org/0000-0002-8147-9388

Universidade do Vale do Taquari, Brasil

E-mail: laura.gaspary@universo.univates.br

\begin{abstract}
Resumo
Introdução: $\mathrm{O}$ uso de organofosforados está presente em todo o mundo na agricultura, indústria e até em ambientes domésticos. Inúmeras pessoas são expostas a esses agrotóxicos, principalmente a população agrícola, que está sujeita aos seus efeitos. Objetivo: realizar uma revisão sistemática da literatura em artigos que avaliaram a exposição crônica a baixas doses de organofosforados em populações rurais. Metodologia: A metodologia utilizada foi a abordagem PICo5 e seu procedimento técnico foi a revisão sistemática da literatura, seguindo as etapas descritas no Preferred Reporting Items for Systematic Reviews and Meta-Analyses (PRISMA). Resultados: As bases dos periódicos consultados, utilizando as palavras-chave "rural populations" e "organophosphates", resultaram em 61 artigos científicos válidos, dos quais 22 atenderam aos critérios de elegibilidade e foram incluídos nessa revisão. Os problemas estudados consideram o tempo de exposição, os tipos de organofosforados, os efeitos na saúde dos trabalhadores e a utilização de Equipamentos de Proteção Individual, e os benefícios do tema estão relacionados a compreender as implicações a exposição crônica a organofosforados, que até hoje carece de estudos. Conclusão: Dentre a clínica mais comumente encontrada, observou-se convergência para efeitos no sistema nervoso central e oncológico decorrentes deste tipo de exposição.
\end{abstract}

Palavras-chave: Organofosforados; Exposição crônica; Populações rurais; Efeitos na saúde; Sistema nervoso central e oncológico.

\begin{abstract}
Introduction: The use of organophosphates is present throughout the world in agriculture, industry and even in domestic environments. Many people are exposed to these pesticides, especially the agricultural population, which is subject to their effects. Objective: to carry out a systematic literature review of articles that evaluated chronic exposure to low doses of organophosphates in rural populations. Methodology: The methodology used was the PICo5 approach and its technical procedure was the systematic literature review, following the steps described in the Preferred Reporting Items for Systematic Reviews and Meta-Analyses (PRISMA). Results: The databases of the journals consulted, using the keywords "rural populations" and "organophosphates", resulted in 61 valid scientific articles, of which 22 met the eligibility criteria and were included in this review. The problems studied consider the exposure time, the types of organophosphates, the effects on workers' health and the use of Personal Protective Equipment, and the benefits of the topic are related to understanding the implications of chronic exposure to organophosphates, which still lacks of studies. Conclusion: Among the most commonly found clinic, there was convergence for effects on the central nervous system and oncologic resulting from this type of exposure.
\end{abstract}


Keywords: Organophosphates; Chronic exposure; Rural populations; Health effects; Central nervous system and cancer.

\section{Resumen}

Introducción: El uso de organofosforados está presente en todo el mundo en la agricultura, la industria e incluso en el ámbito doméstico. Muchas personas están expuestas a estos plaguicidas, especialmente la población agrícola, que está sujeta a sus efectos. Objetivo: realizar una revisión bibliográfica sistemática de artículos que evaluaran la exposición crónica a bajas dosis de organofosforados en poblaciones rurales. Metodología: La metodología utilizada fue el enfoque PICo5 y su procedimiento técnico fue la revisión sistemática de la literatura, siguiendo los pasos descritos en los Ítems Preferidos de Reportes para Revisiones Sistemáticas y Metanálisis (PRISMA). Resultados: Las bases de datos de las revistas consultadas, utilizando las palabras clave "poblaciones rurales" y "organofosforados", arrojaron 61 artículos científicos válidos, de los cuales 22 cumplieron los criterios de elegibilidad y fueron incluidos en esta revisión. Los problemas estudiados consideran el tiempo de exposición, los tipos de organofosforados, los efectos en la salud de los trabajadores y el uso de Equipos de Protección Personal, y los beneficios del tema se relacionan con la comprensión de las implicaciones de la exposición crónica a organofosforados, que aún carece de estudios. Conclusión: Entre las clínicas más comúnmente encontradas, hubo convergencia de los efectos sobre el sistema nervioso central y el cáncer resultante de este tipo de exposición.

Palabras clave: Organofosforados; Exposición crónica; Poblaciones rurales; Efectos en la salud; Sistema nervioso central y oncología.

\section{Introdução}

O grupo de compostos químicos organofosforados (OFs) é amplamente utilizado em todo o mundo, sua aplicabilidade alcança as mais diversas áreas, estando presente na agricultura, indústria e até em ambientes domésticos. Atualmente, durante a vida, há grandes chances de inúmeras pessoas serem expostas a esses produtos químicos (Mackenzie et al., 2010).

A exposição cada vez mais frequente a agrotóxicos, principalmente dos trabalhadores rurais, tem gerado um aumento nas preocupações referentes à saúde da população em geral. Nesse sentido, há evidências científicas que apresentam sua forte relação com o desenvolvimento de algumas patologias, tais como: diferentes tipos de câncer, diabetes, doenças neurodegenerativas (Parkinson, Alzheimer e esclerose lateral amiotrófica), transtornos psiquiátricos, alterações congênitas e distúrbios reprodutivos (Mostafalou, Abdollahi, 2013).

O uso extensivo de OFs na agricultura e em aplicações domésticas levantam questões sobre a segurança da exposição a longo prazo, pois além da frequência, o nível de exposição apresenta-se como outro parâmetro importante a ser observado. Embora existam evidências que corroboram os efeitos na saúde humana da intoxicação aguda por organofosforados, as consequências da exposição de longo prazo a baixas doses destes pesticidas carecem de mais estudos (Committee on Toxicity of Chemicals, 2004).

Além disso, a exposição não se limita aos agricultores que misturam ou aplicam diretamente os pesticidas, mas também inclui suas famílias, comerciantes de agrotóxicos e residentes próximos às áreas de cultivo. Dessa forma, essas populações agrícolas estão sujeitas tanto ocupacionalmente quanto ambientalmente aos efeitos dos agrotóxicos, oportunizando uma exposição crônica (Serrano-Medina et al., 2019).

Nesse contexto, o objetivo deste trabalho é avaliar, por meio de uma revisão sistemática da literatura, se a exposição crônica de populações rurais a baixas doses de organofosforados tem efeitos na sua saúde a longo prazo.

\section{Metodologia}

\subsection{Delineamento e registro do protocolo}

A partir da hipótese elaborada com a metodologia PICo (Moola et al., 2017), voltada para pesquisas não clínicas, protocolou-se o estudo na plataforma International Prospective Register of Systematic Reviews (PROSPERO), cujo registro ocorreu em outubro de 2020 sob o ID CRD42020208965. Toda a documentação dos resultados encontrados durante a revisão sistemática da literatura seguiu de acordo com as etapas descritas no Preferred Reporting Items for Systematic Reviews and 
Meta-Analyses (PRISMA) (Galvão, Pansani \& Harrad 2015).

\subsection{Critérios de elegibilidade}

Foram incluídas publicações de estudos observacionais (estudos longitudinais/coorte, transversais/prevalência e casocontrole) que avaliassem a associação entre a exposição crônica a baixas doses de organofosforados em populações rurais. Todas as etnias e faixas etárias foram incluídas.

Foram excluídos estudos em que não se teve acesso ao artigo completo, revisões sistemáticas, revisões de literatura ou outros tipos de estudos, que abordavam outras populações (não rurais), que traziam resultados inespecíficos para exposição aos organofosforados ou que relatavam exposição a outras classes de pesticidas, que traziam efeitos agudos na saúde, e que o desfecho não era específico para saúde dos indivíduos.

\subsection{Fontes de informação e estratégias de busca}

A metodologia PICo (Moola et al., 2017) permitiu a construção dos descritores para as buscas bibliográficas nas bases de dados: PubMed (PM), Medical Literature Analysis and Retrieval System Online (MEDLINE), Excerpta Medica dataBASE (EMBASE), Scientific Electronic Library Online (SciELO), Biblioteca Cochrane, Biblioteca Virtual em Saúde (BVS) e Literatura Latino-Americana e do Caribe em Ciências da Saúde (LILACS).

A estratégia de busca foi construída por um pesquisador e revisada, de forma independente, por outro pesquisador. As buscas foram atualizadas até 19 de setembro de 2020. Não houve restrições para idade dos participantes, idioma, status e ano de publicação. Utilizou-se a seguinte estratégia de busca no banco de dados PubMed, sendo, posteriormente, adaptada para as outras plataformas: (((c"rural populations" [mesh] [all fields] OR Population [mesh] [all fields] OR Rural [mesh] [all fields] OR "Populations Rural" [mesh] [all fields] OR "Rural Spatial Distribution" [mesh] [all fields] OR Distribution [mesh] [all fields] OR Distributions [mesh] [all fields] OR "Rural Communities" [mesh] [all fields] OR "Rural Community" [mesh] [all fields]) AND (Organophosphates [mesh][all fields] OR Organopyrophosphates [mesh] [all fields] OR "Phosphoric Acid Esters" [mesh] [all fields] OR Esters [mesh] [all fields] OR "Phosphoric Acid" [mesh] [all fields] OR "Organic Phosphates") [mesh] [all fields] AND ("Occupational Exposure" [mesh][all fields] OR "Occupational Exposures" [mesh] [all fields])))).

Adicionalmente, foram examinadas as listas de referências bibliográficas dos estudos relevantes para identificar outros artigos potencialmente elegíveis.

\subsection{Processo de coleta dos dados}

Os artigos incluídos no estudo foram selecionados por dois autores (FW e JK). Em caso de dúvida, o desempate era realizado pelo terceiro autor (CR). Inicialmente, para a seleção dos estudos e extração dos dados, utilizou-se o sistema Rayyan (Ouzzani et al., 2016). Após a remoção dos estudos duplicados, os dois revisores selecionaram os artigos de forma independente. A seleção foi realizada conforme os critérios de elegibilidade pré-definidos, considerando inicialmente o título do estudo e, após, o resumo.

Os artigos selecionados foram lidos na íntegra, também de forma independente, pelos dois autores. A concordância entre os revisores foi alta (Kappa $=0,83$ ). Em todas as etapas, os casos de discordância foram resolvidos por consenso entre os revisores, ou pelo terceiro revisor. Os artigos que não continham características da população estudada foram excluídos.

Os seguintes dados foram extraídos: autor, ano de publicação, país, delineamento do estudo, tipos de organofosforados, tamanho da amostra, fontes de financiamento e desfechos. 


\subsection{Avaliação da qualidade metodológica}

A qualidade metodológica e o risco de vieses dos estudos selecionados foram avaliados por dois pesquisadores, de forma independente, utilizando-se de protocolos específicos para cada tipo de estudo observacional.

A escala de Loney et al. (1998), adaptada para o presente estudo, avaliou as publicações sobre prevalência. Quando um parâmetro era cumprido, o estudo recebia um ponto. Considerou-se de alta qualidade os estudos que pontuaram entre 7 e 8 ; de moderada qualidade àqueles com $4 \mathrm{a} 6$; e de baixa qualidade 0 a 3 pontos.

A ferramenta do Instituto Joanna Briggs (Moola et al., 2017) avaliou os estudos de coorte e caso-controle. Quando um critério era cumprido, o estudo recebia um ponto. Considerou-se de alta qualidade os estudos que pontuaram 10 e 11; moderada qualidade àqueles com 5 a 9; e de baixa qualidade de 0 a 4 pontos.

De maneira geral, a qualidade metodológica dos estudos foi avaliada como moderada. As publicações de prevalência, por vezes, não foram claras quanto às características populacionais. Os estudos de coorte incluídos, por vezes, não foram claros quanto às informações a respeito das variáveis confundidoras e grupos comparáveis. Nenhum estudo foi excluído pela qualidade metodológica.

\subsection{Síntese dos resultados e análises estatísticas}

Os desfechos avaliados na presente revisão sistemática foram: tempo de exposição, tipos de organofosforados, efeito na saúde dos trabalhadores e utilização de Equipamentos de Proteção Individual (EPIs). Os resultados dos estudos incluídos foram apresentados de forma descritiva.

Os estudos incluídos tiveram seus desfechos avaliados considerando algumas estimativas de tamanho de efeito (diferença de risco absoluto, risco relativo, razão de chances, entre outros) e, também, suas respectivas medidas de confiança e variância (intervalos de confiança, medida de dispersão e valores de p). Devido ao alto grau de heterogeneidade dos estudos incluídos, não foi possível conduzir uma metanálise entre os mesmos.

\section{Resultados}

\subsection{Seleção dos estudos}

A Figura 1 descreve o processo de seleção de artigos. As buscas nas bases de dados resultaram um total de 347 trabalhos, sendo que 19 duplicatas foram excluídas imediatamente ao início da revisão. Foram rastreadas pelo título e resumo 328 referências, das quais 267 foram excluídas por não atenderem aos critérios de elegibilidade. Após as avaliações iniciais, 61 artigos tiveram seu texto lido por completo, dos quais 22 foram incluídos nesta revisão sistemática. Nenhum estudo foi incluído na análise após exame das listas de referências dos artigos revisados. 
Figura 1. Fluxograma de seleção dos artigos sobre a exposição de populações rurais aos pesticidas organofosforados.

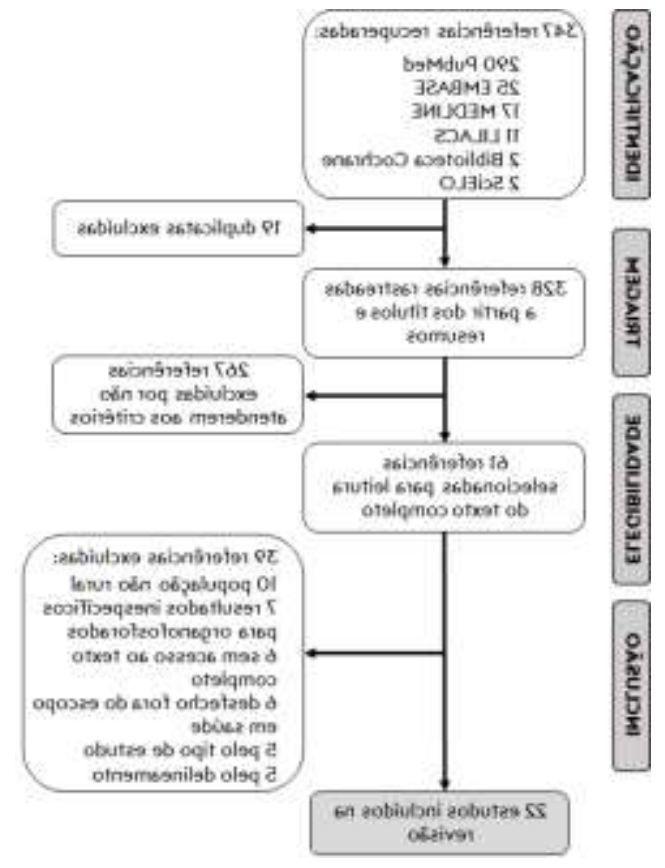

Fonte: Dados da pesquisa (2021).

\subsection{Principais características dos estudos incluídos}

A identificação e as principais características dos estudos incluídos estão apresentadas nas Tabelas 1 e 2 . Dentre os 22 estudos incluídos, a maioria eram transversais e caso-controles e foram conduzidos nos Estados Unidos. A maior parte dos estudos foi publicada entre os anos de 2006 e 2015.

A idade mínima das populações estudadas foi 18 anos e máxima 60 anos. Nas publicações, o tempo de exposição dos trabalhadores rurais variou entre meses e décadas, sendo o mínimo três meses e o máximo quarenta e cinco anos de trabalho exposto aos compostos organofosforados. Indivíduos de sexo masculino são os mais envolvidos nos estudos. O uso de EPIs não foi informado em 12 estudos. A maioria dos demais estudos demonstraram que os trabalhadores não faziam o uso, variando de $62,4 \%$ até $100 \%$ a frequência de trabalhadores que não se protegem ao manipular os pesticidas. Apenas um estudo identificou o uso dos EPIs em 96,2\% dos participantes. 
Tabela 1. Identificação dos artigos selecionados.

\begin{tabular}{|c|c|c|c|c|c|}
\hline Autor & Ano & País & Delineamento & nostra & Fontes de financiamento \\
\hline Alavanja et al. & 2004 & $\begin{array}{l}\text { Estados } \\
\text { Unidos }\end{array}$ & Coorte prospectivo & 57.284 & Não informado \\
\hline Bayrami et al. & 2011 & Irã & Caso-controle & 80 & Tabriz University \\
\hline Cataño et al. & 2007 & Peru & Transversal & 213 & Não informado \\
\hline Engel et al. & 2014 & $\begin{array}{l}\text { Estados } \\
\text { Unidos }\end{array}$ & Transversal & 136 & Não informado \\
\hline Eskenazi et al. & 2007 & $\begin{array}{l}\text { Estados } \\
\text { Unidos }\end{array}$ & Coorte prospectivo & 531 & Sem financiamento \\
\hline Fareed et al. & 2013 & Índia & Caso-controle & 243 & Indian Institute of Toxicology Research \\
\hline Fieten et al. & 2009 & Costa Rica & Transversal & 127 & Não informado \\
\hline $\begin{array}{l}\text { Ghafouri-Khosrowshahi } \\
\text { et al. }\end{array}$ & 2019 & Irã & Caso-controle & 60 & Sem financiamento \\
\hline Hayden et al. & 2010 & $\begin{array}{l}\text { Estados } \\
\text { Unidos }\end{array}$ & Coorte prospectivo & 3.084 & Não informado \\
\hline Hoshino et al. & 2008 & Brasil & $\begin{array}{l}\text { Coorte retrospectivo e } \\
\text { Transversal }\end{array}$ & 18 & Não informado \\
\hline Jones et al. & 2015 & $\begin{array}{l}\text { Estados } \\
\text { Unidos }\end{array}$ & Coorte prospectivo & 22.830 & 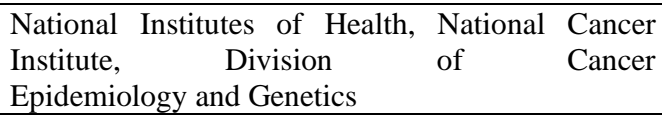 \\
\hline Lerro et al. & 2015 & $\begin{array}{l}\text { Estados } \\
\text { Unidos }\end{array}$ & Coorte prospectivo & 30.003 & $\begin{array}{l}\text { Intramural Research Program of the } \\
\text { National Institutes of Health, the National Cancer } \\
\text { Institute and the } \\
\text { National Institute of Environmental Health Sciences }\end{array}$ \\
\hline Muñoz-Quezada et al. & 2016 & Chile & Transversal & 177 & $\begin{array}{l}\text { Fundo Nacional de Pesquisa em Saúde da Comissão } \\
\text { Nacional de Ciência e Tecnologia do Chile, FONIS } \\
\text { e pela Universidade Católica del Maule. }\end{array}$ \\
\hline Mackenzie Ross et al. & 2010 & Inglaterra & $\begin{array}{l}\text { Coorte retrospectivo e } \\
\text { Caso-controle }\end{array}$ & 127 & Não informado \\
\hline Rothlein et al. & 2006 & $\begin{array}{l}\text { Estados } \\
\text { Unidos } \\
\end{array}$ & Caso-controle & 141 & Sem financiamento \\
\hline Salem et al. & 2020 & Egito & Caso-controle & 51 & Sem financiamento \\
\hline Salvi et al. & 2003 & Brasil & Transversal & 25 & $\begin{array}{l}\text { CNPq, PRONEX e Pontifícia Universidade Católica } \\
\text { do Rio Grande do Sul (PUCRS). }\end{array}$ \\
\hline Serrano-Medina et al. & 2019 & México & $\begin{array}{l}\text { Caso-controle } \\
\text { transversal }\end{array}$ & 240 & $\begin{array}{l}\text { Universidad Autonóma de Baja California, 19th and } \\
\text { 20th Convocatoria Interna and SEP-PRODES } \\
\text { Support for the Strengthening of Academic Bodies }\end{array}$ \\
\hline $\begin{array}{l}\text { Shomar; } \quad \text { Al-Saad; } \\
\text { Nriagu }\end{array}$ & 2014 & Qatar & Transversal & 125 & $\begin{array}{l}\text { Qatar National Research Fund (a member of The } \\
\text { Qatar } \\
\text { Foundation) }\end{array}$ \\
\hline Stokes et al. & 1995 & $\begin{array}{l}\text { Estados } \\
\text { Unidos } \\
\end{array}$ & $\begin{array}{lll}\begin{array}{l}\text { Coorte } \\
\text { controle }\end{array} & \text { e Caso- } \\
\end{array}$ & 158 & Não informado \\
\hline Tahmaz et al. & 2003 & $\begin{array}{l}\text { Reino } \\
\text { Unido }\end{array}$ & $\begin{array}{l}\text { Coorte retrospectivo e } \\
\text { Caso-controle }\end{array}$ & 63 & Karim Rida Said Foundation \\
\hline Zahm et al. & 1993 & $\begin{array}{l}\text { Estados } \\
\text { Unidos } \\
\end{array}$ & Caso-controle & 590 & Não informado \\
\hline
\end{tabular}

Fonte: Dados da pesquisa (2021). 
Tabela 2. Distribuição das principais características dos estudos.

\begin{tabular}{lcc}
\hline VARIÁVEIS & (n) & \% \\
\hline Ano de publicação & & \\
1993 a 2000 & 2 & 9,1 \\
2001 a 2005 & 3 & 13,6 \\
2006 a 2010 & 7 & 31,8 \\
2011 a 2015 & 6 & 27,3 \\
2016 a 2020 & 4 & 18,2 \\
Tamanho da amostra & & \\
até 50 & 2 & 9,1 \\
51 a 100 & 4 & 18,2 \\
101 a 500 & 10 & 45,5 \\
501 a 1000 & 2 & 9,1 \\
1001 a 10.000 & 1 & 4,5 \\
10.001 a 50.000 & 2 & 9,1 \\
50.001 ou mais & 1 & 4,5 \\
Sexo & & \\
Todos masculino & 8 & 36,4 \\
Maioria masculino & 6 & 27,3 \\
Todos feminino & 4 & 18,2 \\
Maioria feminino & 3 & 13,6 \\
Não especifica & 1 & 4,5 \\
Local do estudo & & \\
África & 1 & 4,5 \\
América do Sul & 4 & 18,2 \\
América do Norte & 11 & 50,0 \\
Ásia & 4 & 18,2 \\
Europa & 2 & 9,1 \\
Qualidade dos estudos & & \\
Alta & 3 & 13,6 \\
Moderada & 18 & 81,8 \\
Baixa & 1 & 4,6 \\
\hline & & \\
\hline & &
\end{tabular}

Fonte: Dados da pesquisa (2021).

\subsection{Categorias de desfechos em saúde}

A partir dos estudos incluídos nesta revisão, observou-se dez afecções em saúde correlacionadas com a exposição crônica aos compostos organofosforados.

\subsection{Sistema respiratório}

Fareed et al. (2013) realizou um estudo caso-controle, no qual 36,75\% dos pulverizadores de organofosforados apresentaram algum sintoma respiratório, sendo tosse seca e dispneia significativamente mais comuns entre os aplicadores de pesticidas em comparação ao grupo controle. Além disso, os pulverizadores que permaneceram mais tempo expostos aos pesticidas exibiram maior prevalência de problemas respiratórios.

Fieten et al. (2009) em estudo transversal, realizou testes de espirometria para obter capacidade vital forçada e volume expiratório forçado em 1 segundo. Entre os expostos, a prevalência de chiado foi de $20 \%$ e de falta de ar foi de $36 \%$ contra $9 \%$ e 26\%, respectivamente, para os não expostos. Os inseticidas clorpirifós e terbufos foram fortemente associados com sibilância. Para ambos inseticidas, foi encontrada uma associação estatisticamente significativa do efeito exposição. Exposição a múltiplos organofosforados era comum; $81 \%$ das mulheres expostas foram expostas a clorpirifós e terbufos. Consequentemente, seus efeitos não poderiam ser separados. Nenhuma relação entre a exposição a pesticidas e função pulmonar ventilatória foi encontrada. A publicação não pôde estabelecer uma relação causal entre a exposição a pesticidas e sintomas respiratórios, mas sugere associações. 


\subsection{Sistema hematopoiético}

Fareed et al. (2013) também demonstrou aumento de alterações hematológicas (leucopenia, neutropenia, monocitose, anemia e trombocitopenia) entre os trabalhadores expostos com queixas respiratórias ao compará-los aos indivíduos sem sintomas respiratórios.

\subsection{Oncológico}

Salem et al. (2020) evidenciou, por meio de um estudo caso-controle, a associação entre exposição aos OFs e a expressão reduzida de mRNA de P53 e P21, que são genes supressores de diversos tumores. Essa expressão reduzida está relacionada com níveis baixos da enzima acetilcolinesterase (AChE), também considerada um marcador de exposição aos OFs. A partir disso, sugeriu que a exposição crônica aos OFs pode ser um risco para o desenvolvimento de câncer em aplicadores de pesticidas.

Jones et al. (2015) realizou um estudo de coorte prospectivo com 22.830 participantes para avaliar a relação entre incidência de tumores sólidos e exposição a agrotóxicos. Após análises multivariáveis, observou uma tendência com significância estatística da incidência de câncer de pulmão com o tempo de exposição ao diazinon, especificamente para o tipo histológico adenocarcinoma. Além disso, após ajuste de fatores confusionais ou potencialmente confundidores durante o acompanhamento, evidenciou riscos igualmente elevados para câncer de rim e de próstata entre os aplicadores ao comparar com o grupo controle.

Zahm et al. (1993), em um estudo caso-controle, verificou que nem o uso de inseticidas nem herbicidas foi associado ao linfoma B não-Hodgkin. Pequenas associações não significativas foram observadas entre as mulheres que tinham contato direto com pesticidas ou herbicidas.

Alavanja et al. (2004) em estudo de coorte prospectivo, examinou a relação entre 50 pesticidas agrícolas amplamente utilizados com a incidência de câncer de pulmão. Uma taxa de incidência padronizada de câncer de pulmão de 0,44 ( $\mathrm{p}=0,05$ : 0,39-0,49) foi observada em geral, devido em grande parte a uma baixa prevalência de tabagismo. Dois herbicidas, metolacloro e pendimetalina, e dois inseticidas - clorpirifós e diazinon, amplamente utilizados,, mostraram alguma evidência de resposta de exposição para câncer de pulmão, mas a tendência foi significativa apenas para clorpirifós. Em conclusão, pelo menos quatro pesticidas amplamente utilizados atualmente nos Estados Unidos e em outros lugares são significativamente associados ao risco de câncer de pulmão.

Lerro et al. (2015) realizou um estudo de coorte prospectivo a fim de verificar a incidência de câncer entre as esposas dos aplicadores de pesticidas. O uso de OFs foi associado a um risco elevado de câncer de mama. Entre 30.003 mulheres, 25,9\% relataram exposição a organofosforados, e destas, 718 mulheres foram diagnosticadas com câncer durante o período de acompanhamento. Os autores observaram um risco aumentado com o uso de OFs para vários tipos de câncer relacionados aos hormônios, incluindo mama, tireóide e ovário.

\subsection{Sistema reprodutor}

Ghafouri-Khosrowshahi et al. (2019) realizou um estudo de caso-controle em uma população rural com maior incidência de infertilidade. Demonstrou que a quantidade e a motilidade espermática em trabalhadores rurais, que utilizam os OFs, foi mais baixa do que no grupo controle não exposto. Segundo seu artigo, os pesticidas clorpirifós, melatião e paratião podem afetar o número de espermatozóides com a proliferação de células germinativas, o que pode causar lesão no epitélio seminífero.

A partir da medida reduzida da capacidade antioxidante total (CAC) encontrada no sêmen, bem como níveis séricos elevados de lipoperoxidação (LPO), confirmou-se a presença de estresse oxidativo no organismo de agricultores pulverizadores de OFs, comprometendo sua produção espermática e função de suas gônadas. Contudo, a diminuição do número e qualidade de espermatozóides não é suficiente para explicar a maior incidência de infertilidade nessa população. 


\subsection{Sistema auditivo}

Hoshino et al. (2008) realizou um estudo transversal com 18 trabalhadores rurais, no qual a queixa de tontura foi relatada por toda a amostra. Tal sintoma clínico é, por vezes, associado à exposição crônica aos OFs. Além disso, 7 (38,9\%) dos aplicadores de agrotóxicos apresentaram alterações auditivas e $16(88,8 \%)$ tiveram comprometimento vestibular.

Outro apontamento do estudo traz a relação do tempo de exposição diária ao agrotóxico com número de alterações nos resultados dos exames realizados, uma vez que oito trabalhadores rurais (44,5\%), que apresentaram alterações de equilíbrio, trabalhavam mais de 21 anos com OFs, e 13 pulverizadores (72,3\%) trabalhavam mais de 10 horas por dia e estavam com alterações na vectoeletronistagmografia (VENG), exame que avalia o sistema vestibular.

\subsection{Sistema nervoso central}

Serrano-Medina et al. (2019) descreveu um estudo caso-controle com 140 aplicadores de OFs, na sua maioria mulheres, e com pelo menos um sintoma neurológico (80\%), sendo que muitas relataram sintomatologia durante a jornada de trabalho nas propriedades rurais. As queixas mais comuns foram: dor nas costas $(64,3 \%)$, dormência de membros $(51,4 \%)$, tontura $(51,4 \%)$, dor nos ombros $(48,6 \%)$, desconforto abdominal $(40,7 \%)$, dispneia $(31,4 \%)$, noctúria $(34,3 \%)$ e insônia $(25,7 \%)$.

Salvi et al. (2003) descreveu que a presença sintomas extrapiramidais, medida pelo escore total de parkinsonismo (ESRS), foi observada em 12 trabalhadores rurais, sendo eles tremor, fácies inexpressiva e movimento pendular de membros superiores reduzidos os mais comumente encontrados. Ao serem avaliados novamente após 3 meses sem exposição aos OFs, 9 trabalhadores permaneceram com escore ESRS elevado, apresentando os sintomas extrapiramidais no retorno, o que foi considerado significativo tendo em vista a faixa etária da população estudada.

Mackenzie Ross et al. (2010) realizou um estudo de coorte retrospectivo e caso-controle para verificar se a exposição a baixo nível de pesticidas organofosforados causa deficiência neurológica ou psiquiátrica. Em termos de saúde emocional e física, mais de $40 \%$ da coorte exposta queixou-se de níveis clinicamente significativos de ansiedade e depressão em comparação com menos de $23 \%$ dos controles, as maiores taxas de angústia foram verificadas nos agricultores aposentados. Os agricultores também relatam uma gama de sintomas físicos que eles descrevem como sendo moderados a grave, sendo o mais proeminente a fadiga, problemas de memória, articulações rigidez, perturbações do sono, irritabilidade e sensação de lentificação do pensamento. Em termos de função cognitiva, capacidade intelectual geral, raciocínio, habilidade visuoespacial e verbal foram relativamente bem preservadas, mas trabalhadores agrícolas obtiveram pontuações mais baixas em testes de velocidade de resposta, trabalho, memória verbal e visual, flexibilidade mental e motora fina controle, do que controles. Os resultados sugerem que pode existir relação entre a exposição de baixo nível a organofosforados e funcionamento neurocomportamental.

Muñoz-Quezada et al. (2016), desenvolveu estudo transversal para avaliar o desempenho neuropsicológico e motor de trabalhadores expostos a organofosforados. Os trabalhadores expostos apresentam desempenho abaixo da média normativa esperada. Idade, sexo e anos de estudo estavam significativamente relacionados às funções neuropsicológicas e neuromotoras, sendo que as variáveis que se mostraram significativas nos modelos foram anos de estudo, idade e sexo. Ajustando os resultados do desempenho neuropsicológico e neuromotor para essas variáveis, observou-se que os expostos desempenharam em média 3 pontos a menos que os não expostos na compreensão verbal, 4 pontos a menos na velocidade de processamento, 4 pontos a menos no QI e mais 1 ponto de dificuldade nos reflexos profundos e na sensibilidade discriminativa.

Rothlein et al. (2006) em estudo caso-controle verificou que o desempenho neurocomportamental de trabalhadores rurais imigrantes hispânicos foi inferior ao observado em uma população de imigrantes hispânicos não agrícolas. Dentre a amostra de trabalhadores agrícolas houve correlação positiva entre níveis de organofosforados urinários, níveis de metabólitos e desempenho inferior em alguns testes neurocomportamentais. Essas descobertas somam-se a um aumento das evidências de 
associação entre baixos níveis de exposição a pesticidas e déficits no desempenho neurocomportamental.

Eskenasi et al. (2007) em estudo de coorte prospectivo, investigou a relação entre o neurodesenvolvimento infantil e os níveis de metabólitos urinários de organofosforados no período pré-natal e infantil. Seis metabólitos de dialquilfosfato (DAPs) inespecíficos foram verificados em urina materna e infantil, bem como metabólitos específicos para malatião (MDA) e clorpirifós (TCPy) na urina materna. Os autores examinaram a relação com o desempenho das crianças aos 6 ( $n=396), 12$ ( $n=395)$ e 24 (n = 372) meses de idade nas Escalas Bayley de Desenvolvimento Infantil, que sugeriram associações entre a presença de DAPs pré-natais com atrasos no desenvolvimento neuropsicomotor.

Hayden et al. (2010) realizou um estudo de coorte prospectivo para verificar o risco de demência e doença de Alzheimer em aplicadores de pesticidas. Os resultados mostraram que havia um risco aumentado de demência em pessoas expostas a qualquer pesticida. O risco de demência associada à exposição a organofosforados após o ajuste para idade, sexo, educação, score de 3MS e o status de ApoE-4 aumentou entre os expostos, embora seja marginalmente não significativo. Estas descobertas adicionam a uma pequena, mas crescente literatura sugerindo que a exposição a pesticidas pode ter efeitos adversos de longo prazo no sistema nervoso, aumentando assim o risco de doença de Alzheimer no final da vida.

\subsection{Sistema nervoso periférico}

Engel et al. (1998) por meio de um estudo transversal com 67 trabalhadores rurais hispânicos, não observou relação estatística entre a exposição de trabalhadores agrícolas aos baixos níveis de organofosforados com disfunção neurofisiológica periférica, durante uma temporada.

Stokes et al. (1995) realizaram um estudo de coorte e caso-controle com 90 aplicadores de pesticidas, a fim de investigar o efeito da exposição aos organofosforados no sistema nervoso periférico. A sensibilidade do limiar de vibração foi usada como um indicador de neurotoxicidade crônica. Um aumento significativo na sensibilidade de limiar de vibração média para a mão dominante e não dominante sugeriu que a exposição anterior a organofosforados entre os aplicadores de pesticidas estava associada a uma perda de função do nervo periférico.

\subsection{Sistema musculoesquelético}

Shomar et al. (2014) realizaram um estudo transversal com 125 pulverizadores de agrotóxicos, evidenciando que o manuseio incorreto dos pesticidas, isto é, aplicação sem EPIs, desconhecimento sobre armazenamento correto de embalagens vazias e descarte de resíduos predispõem os trabalhadores às intoxicações agudas e crônicas. Durante a pesquisa, os sintomas mais comumente relatados pelos trabalhadores rurais foram: rigidez e dor articular, cefaléia frequente e sudorese excessiva. Os seus resultados de biomonitoramento demonstraram níveis séricos detectáveis de metabólitos alquifos nas amostras coletadas, bem como os compostos metabolizados dietilalquilfosfatos (DEAP) e dimetilalquilfosfatos (DMAP) na urina desses trabalhadores, os quais são considerados indicadores de exposição aos OFs.

\subsection{Transtornos mentais}

Serrano-Medina et al. (2019) obtiveram diferenças estatísticas significativas no diagnóstico de transtornos psiquiátricos entre o grupo exposto aos pesticidas e o não exposto, visto que 31,4\% das agricultoras expostas tinham diagnóstico de depressão maior com risco de suícidio. Por outro lado, $25,7 \%$ das pulverizadoras não apresentaram nenhum transtorno psiquiátrico.

Salvi et al. (2003), por meio de um estudo transversal, avaliaram a incidência de transtornos psiquiátricos e comprometimento neurológico com a exposição a OFs. No artigo, entre os 25 participantes que completaram as avaliações de saúde mental (durante e após exposição), inicialmente, 18 (48\%) apresentaram diagnóstico de ansiedade generalizada e 8 (21\%) de episódios de depressão maior. 


\subsection{De natureza sistêmica}

Bayrami et al. (2012) discutiram sobre a produção de radicais livres e estresse oxidativo na população exposta aos OFs. Em seus resultados houve aumento da atividade da enzima superóxido dismutase (SOD) e incremento nas concentrações séricas de lipoperoxidação (LPO). Ao comparar com o grupo controle, os agricultores estavam com o nível de capacidade antioxidante total (CAT) e atividade da enzima acetilcolinesterase (AChe) diminuídos, o que corrobora para a existência de intoxicação e absorção dos pesticidas pelo organismo. Durante a pesquisa, os pulverizadores de OFs apresentaram sintomas clínicos como: eczema na pele, dispnéia, náuseas e salivação excessiva.

Tahmaz et al. (2003) apresentaram resultados de um estudo de caso-controle, cujos trabalhadores rurais relataram sintomas condizentes com a síndrome da fadiga crônica (SFC) após exposição aos OFs. Tal síndrome é caracterizada por intensa fadiga, dificuldade em realizar atividades físicas e cognitivas e mal-estar, sendo, geralmente, exacerbada após esforço físico ou mental.

Cataño et al. (2008), em estudo transversal, verificaram os níveis de PChE (colinesterase plasmática) em dois subgrupos expostos (aplicadores de pesticidas e outros empregos agrícolas). Aproximadamente $61 \%$ dos trabalhadores expostos relataram sintomas relacionados a pesticidas, mas sem diferença significativa em comparação aos trabalhadores sem sintomas. Por outro lado, o uso de EPI foi significativamente associado a níveis mais elevados de PChE e a um menor risco de relatar sintomas relacionados a pesticidas, o que apoia o benefício da utilização de medidas de proteção adequadas.

\section{Discussão}

A maioria dos estudos incluídos nesta revisão descreveu taxas de prevalência e incidência de efeitos na saúde das populações rurais expostas aos organofosforados. Foram identificados estudos de cinco continentes, com amplo espectro de desfechos em saúde, os quais utilizaram variados critérios de inclusão, métodos de rastreio, investigação e diagnóstico. Durante a análise dos conteúdos das publicações, elencou-se dez afecções em saúde decorrentes da exposição crônica aos compostos organofosforados.

Entre os artigos selecionados, a maioria era transversal e caso-controle. A partir da clínica e resultados de exames complementares das populações estudadas, dentre os mais comumente encontrados, observou-se convergência para efeitos no sistema nervoso central e oncológicos decorrentes deste tipo de exposição.

Os tipos de organofosforados utilizados pelos trabalhadores foram discriminados em 16 estudos, enquanto que os demais não traziam esse tipo de especificação. Os nomes mais citados nos estudos foram, respectivamente: Clorpirifós (11), Malatião (6), Diazinon (5), Paratião (5), 2,2 diclorovinil dimetil fosfato (3), Terbufos (3), Fosmete (3), Metafós (2), Monocrotofós (1), Tamaron 1, Acefato (1), Guthion (1), Fosfamidon (1), Metrifonato ou triclorfon (1), Dimetoato (1) e Azinfosetil (1).

De acordo com os estudos selecionados, o tempo de exposição dos trabalhadores rurais foi maior que cinco anos em sua maioria. Há estudos (Fareed et al., 2013; Hoshino et al., 2008) que sugerem uma relação de causa e efeito entre tempo de exposição e número de alterações patológicas na saúde dos trabalhadores rurais. Os resultados das publicações evidenciaram a infrequência no uso de EPIs durante a manipulação e aplicação de agrotóxicos.

Uma alta heterogeneidade entre os estudos $\left(\mathrm{I}^{2}>75 \%\right)$ foi observada entre os artigos e se manteve em todas as análises de subgrupos. Além de causas metodológicas, a grande heterogeneidade entre os resultados das publicações pode ter ocorrido devido às desigualdades sociais, diferença de aspectos culturais, variação das regulamentações ambientais e sanitárias, bem como listas de produtos químicos aprovados para comercialização e utilização nesses países. 
As prevalências mais baixas de desfechos negativos à saúde nos estudos podem ser atribuídas ao uso correto dos EPIs (Cataño et al., 2008) durante as jornadas de trabalho, manuseio e descarte correto de embalagens e resíduos (Shomar, Al-Saad \& Nriagu, 2014), assim como educação e treinamento dos trabalhadores rurais e comerciantes de organofosforados para adequada manipulação dessa categoria de produtos (Shomar, Al-Saad \& Nriagu, 2014; Cataño et al., 2008).

Uma possível limitação do presente estudo encontra-se na aplicação das escalas de Loney et al. (Loney et al., 1998) e ferramenta do Instituto Joanna Briggs (Moola et al., 2017) para avaliar a qualidade metodológica dos estudos incluídos, uma vez que a atribuição de pesos iguais em cada item pode gerar vieses pela ausência de possível comparação com um parâmetro padrãoouro. Outra limitação aparente, refere-se à avaliação e síntese de evidências, uma vez que não foi possível aplicar-se a abordagem The Grading of Reccomendations Assessment, Development and Evaluation (GRADE) (Guyatt et al., 2011) devido ao alto grau de heterogeneidade entre os estudos incluídos.

\section{Conclusão}

Apesar da variedade de desfechos em saúde, as evidências científicas ainda são preliminares e de moderada qualidade metodológica. O presente estudo aponta e reafirma a importância de pesquisas na área, visto que há um limitado número de trabalhos que abordam o tema, especialmente, tratando-se em exposição crônica. Além disso, em vistas da contínua expansão da cartilha de agrotóxicos aprovados para uso em nosso país, sugere-se que futuros estudos contemplem outras classes de pesticidas.

\section{Referências}

Alavanja, M. C. R., Dosemeci, M., Samanic, C., Lubin, J., Lynch, C. F., Knottt, C., Barket, J., Hoppin, J. A., Sandler, D. P., Coble, J., Thomas, K., \& Blair, A. (2004). Pesticides and lung cancer risk in the agricultural health study cohort. Am J Epidemiol. 160(9):876-885. 10.1093/aje/kwh290

Bayrami, M., Hashemi, T., Malekirad, A. A., Ashayeri, H., Faraji, F., \& Abdollahi, M. (2012). Electroencephalogram, cognitive state, psychological disorders, clinical symptom, and oxidative stress in horticulture farmers exposed to organophosphate pesticides. Toxicol Ind Health. 28(1):90-96. $10.1177 / 0748233711407243$

Cataño, H. C., Carranza, E., Huamaní, C., \& Hernández, A. F. (2008). Plasma cholinesterase levels and health symptoms in peruvian farm workers exposed to organophosphate pesticides. Arch Environ Contam Toxicol. 55(1):153-159. 10.1007/s00244-007-9095-0

Committee on Toxicity of Chemicals (COT) in Food, Consumer Products and the Environment (Department of Health). Organophosphates. Crown Copyright, 2004.

Engel, L. S., Keifer, M. C., Checkoway, H., Robinson, L. R., \& Vaughan, T. L. (1998). Neurophysiological function in farm workers exposed to organophosphate pesticides. Arch Environ Health. 53(1):7-14. 10.1080/00039899809605684

Eskenazi, B., Marks, A. R., Bradman, A., Harley, K., Barr, D. B., Johnson, C. Morga, N. \& Jewell, N. P. (2007). Organophosphate pesticide exposure and neurodevelopment in young Mexican-American children. Environ Health Perspect. 115(5):792-798. 10.1289/ehp.9828

Fareed, M., Pathak, M. K., Bihari, V., Kamal, R., Srivastava, A. K., \& Kesavachandran, C. N. (2013). Adverse respiratory health and hematological alterations among agricultural workers occupationally exposed to organophosphate pesticides: a cross-sectional study in North India. PloS one 8(8):10.1371 10.1371/journal.pone.0069755

Fieten, K. B., Kromhout, H., Heederik, D. J. J, \& Van Wendel de Joode, B. (2009). Pesticide exposure and respiratory health of indigenous women in Costa Rica. Am J Epidemiol. 169(12):1500-1506. 10.1093/aje/kwp060

Galvão, T. F., Pansani, T. S. A., Harrad, D. (2015). Principais itens para relatar revisões sistemáticas e meta-análises: a recomendação PRISMA. Epidemiol Serv Saude. 24(2): 335-342. 10.5123/S1679-49742015000200017

Ghafouri-Khosrowshahi, A., Ranjbar, A., Mousavi, L., Nili-Ahmadabadi, H., Ghaffari, F., Zeinvand-Lorestani, H., \& Nili-Ahmadabadi, A. (2019). Chronic exposure to organophosphate pesticides as an important challenge in promoting reproductive health: A comparative study. J Educ Health Promot. 8:149. 10.4103/jehp.jehp_148_19

Guyatt, G. H., Oxman, A.D., Schünemann, H. J., Tugwell, P., \& Knottnerus, A. (2011). GRADE guidelines: a new series of articles in the Journal of Clinical Epidemiology. J Clin Epidemiol. 64(4):380-382. 10.1016/ j.jclinepi.2010.09.011

Hayden, K. M., Norton, M. C., Darcey, D., Østbye, T., Zandi, P. P., Breitner, J. C. S., \& Welsh-Bohmer, K. (2010) Occupational exposure to pesticides increases the risk of incident AD: the Cache County study. Neurology. 74(19):1524-1530. 10.1212/WNL.0b013e3181dd4423

Hoshino, A. C. H., Ferreira, H. P., Taguchi, C. K., Tomita, S., \& Miranda, M. F. (2008). Estudo da ototoxicidade em trabalhadores expostos a organofosforados. Rev. Bras. Otorrinolaringol. 74(6). doi.org/10.1590/S0034-72992008000600015 
Jones, R. R., Barone-Adesi, F., Koutros, S., Lerro, C. C., Blair, A., Lubin, J., Heltshe, S. L., Hoppin, J. A., Alavanja, M. C. R., \& Freeman L. B. (2015). Incidence of solid tumours among pesticide applicators exposed to the organophosphate insecticide diazinon in the Agricultural Health Study: an updated analysis. Occup Environ Med. 72(7):496-503. 10.1136/oemed-2014-102728

Lerro, C. C., Koutros, S., Andreotti, G., Friesen, M. C., Alavanja, M. C., Blair, A., Hoppin, J. A., Sandler, D. P., Lubin, J. H., Ma, X., Zhang, Y., \& Freeman, L. B. (2015). Organophosphate insecticide use and cancer incidence among spouses of pesticide applicators in the Agricultural Health Study. Occup Environ Med. 72(10):736-744. 10.1136/oemed-2014-102798.

Loney, P. L., Chambers, L. W., Bennett, K. J., Roberts, J. G., \& Stratford, P. W. (1998). Critical appraisal of the health research literature: prevalence or incidence of a health problem. Chronic Dis Can. 19(4):170-176.

Ross, S. J. M., Brewin, C. R., Curran, H. V., Furlong, C. E., Abraham-Smith, K. M., \& Harrison, V. (2010). Neuropsychological and psychiatric functioning in sheep farmers exposed to low levels of organophosphate pesticides. Neurotoxicol Teratol. 32(4):452-459. 10.1016/j.ntt.2010.03.004

Moola, S., Munn, Z., Tufanaru, C., Aromataris, E., Sears, K., Sfetcu, R., Currie, M., Lisy, K., Qureshi, R., Mattis, P., \& Mu, P. (2017). Chapter 7: Systematic reviews of etiology and risk. In: Aromataris, E., Munn, Z. (editors). Joanna Briggs Institute Reviewer's Manual. The Joanna Briggs Institute https://reviewersmanual.joannabriggs.org/

Mostafalou, S., \& Abdollahi, M. (2013). Pesticides and human chronic diseases: evidences, mechanisms, and perspectives. Toxicol Appl Pharmacol. 268(2):157177. 10.1016/j.taap.2013.01.025

Muñoz-Quezada, M. T., Lucero, B., Iglesias, V., Muñoz, M. P., Achú, E., Carnejo, C., Concha, C., Grillo, A., \& Brito, A. M. (2016). Plaguicidas organofosforados y efecto neuropsicológico y motor en la Región del Maule, Chile. Gaceta Sanitaria. 10.1016/j.gaceta.2016.01.006

Ouzzani, M., Hammady, H., Fedorowicz, Z., \& Elmagarmid, A. (2016). Rayyan-a web and mobile app for systematic reviews. Syst Rev. 5(1):210. $10.1186 / \mathrm{s} 13643-016-0384-4$

Rothlein, J., Rohlman, D., Lasarev, M., Phillips, J., Muniz, J., \& McCauley, L. (2006). Organophosphate pesticide exposure and neurobehavioral performance in agricultural and non-agricultural Hispanic workers. Environ Health Perspect. 114(5):691-696. 10.1289/ehp.8182

Salem, E. A., Elhalafawy, I. A., Hegazy, M. M., Younis, F. E., Swellim, O. A., \& Sakr, M. A. (2020). Altered tumor suppressor genes expression in Egyptian pesticide applicators exposed to organophosphate insecticides. Toxicology and industrial health. 36(8):558-566. 10.1177/0748233720940538

Salvi, R. M., Lara, D. R., Ghisolfi, E. S., Portela, L. V., Dias, R. D., \& Souza, D. O. (2003). Neuropsychiatric evaluation in subjects chronically exposed to organophosphate pesticides. Toxicol Sci. 72(2):267-271. 10.1093/toxsci/kfg034

Serrano-Medina, A., Ugalde-Lizárraga, A., Bojorquez-Cuevas, M. S, Garnica-Ruiz, J., González-Corral, M. A., García-Ledezma, A., Pineda-García, G., \& Cornejo-Bravo, J. M. (2019). Neuropsychiatric Disorders in Farmers Associated with Organophosphorus Pesticide Exposure in a Rural Village of Northwest México. International Journal of Environmental Research and Public Health. 16(5): 689. 10.3390/ijerph16050689.

Shomar, B., Al-Saad, K., \& Nriagu, J. (2014). Mishandling and exposure of farm workers in Qatar to organophosphate pesticides. Environ Res. 133:312-320. 10.1016/j.envres.2014.06.010

Stokes, L., Stark, A., Marshall, E., \& Narang, A. (1995). Neurotoxicity among pesticide applicators exposed to organophosphates. Occup Environ Med. 52(10):648-653. 10.1136/oem.52.10.648

Tahmaz, N., Soutar, A., \& Cherrie, J. W. (2003). Chronic fatigue and organophosphate pesticides in sheep farming: a retrospective study amongst people reporting to a UK pharmacovigilance scheme. Ann Occup Hyg. 47(4):261-267. 10.1093/annhyg/meg042

Zahm, S. H., Weisenburger, D. D., Saal, R. C., Vaught, J. B., Babbitt, P. A., \& Blair, A. (1993). The role of agricultural pesticide use in the development of nonHodgkin's lymphoma in women. Arch Environ Health. 48(5):353-358. 10.1080/00039896.1993.9936725 